\title{
Measurements of Steady Flow through a Bileaflet Mechanical Heart Valve using Stereoscopic PIV
}

Chris Hutchison

\author{
Dept. of Mechanical and Industrial Engineering \\ University of Toronto \\ 5 King's College Rd. \\ Toronto, Ontario, Canada M5S $3 G 8$ \\ chris.hutchison@utoronto.ca \\ Pierre Sullivan \\ Dept. of Mechanical and Industrial Engineering \\ University of Toronto \\ 5 King's College Rd. \\ Toronto, Ontario, Canada M5S $3 G 8$ \\ sullivan@mie.utoronto.ca \\ C. Ross Ethier \\ Dept. of Bioengineering \\ Imperial College, London \\ South Kensington Campus, London, UK SW7 2AZ \\ r.ethier@imperial.ac.uk
}

\begin{abstract}
Computational modeling of bileaflet heart valve (BiMHV) flow requires experimentally validated datasets and improved knowledge of BiMHV fluid mechanics. In this study, flow was studied downstream of a model BiMHV in an axisymmetric aortic sinus using stereoscopic particle image velocimetry. The inlet flow was steady and the Reynolds number based on the aortic diameter was 7600 . Results showed the outof-plane velocity was of similar magnitude as the transverse velocity. Although additional studies are needed for confirmation, analysis of the out-ofplane velocity showed the possible presence of a four-cell streamwise vortex structure in the mean velocity field. Spatial data for all six Reynolds stress components were obtained. Reynolds normal stress profiles revealed similarities between the central jet and free jets. These findings are important to BiMHV flow modeling, though clinical relevance is limited due to the idealized conditions chosen. To this end, the dataset is publicly available for CFD validation purposes.
\end{abstract}

Keywords: bileaflet mechanical heart valve, stereoscopic particle image velocimetry, $C F D$ validation, $3 D$ vortex structure, Reynolds stress

\section{Introduction}

The most recent mechanical valve prosthesis in widespread use is the bileaflet mechanical heart valve (BiMHV) [24]. Computational fluid dynamics (CFD) modeling of BiMHV flow has enormous potential in future device improvement because of the ability to perform inexpensive parametric studies. Recently, advanced codes have been developed to model the complex, turbulent, and three-dimensional flow downstream of a BiMHV throughout the entire cardiac cycle $[3,5,6,11,13,26]$. For accurate CFD simulations of BiMHV flow, rigorous code validation is required. Numerical studies of BiMHV flow have found that large scale flow structures break down to smaller scale turbulence near peak 
systole [5]. These small-scale structures have proved difficult to experimentally validate in a pulsatile flow regime due to cycle-to-cycle variability thought to be caused by asynchronous valve leaflet opening, and valve geometry imperfections in-vitro [4,5]. Because of these issues in validation, in initial stages of code development, it is advantageous to validate CFD code at a peak systolic Reynolds number with steady inflow conditions The primary goal of the current study was to facilitate further CFD code development by obtaining an experimental dataset of BiMHV flow with steady inlet conditions, and making it publicly available for validation purposes. The results of the current study were thus limited to the steady inflow condition, where the effects of pulsatile flow acceleration and deceleration, and resulting vortex interaction, were not present. While this limits the clinical relevance of the study, it is well suited for CFD validation purposes.

Yoganathan et al. [31,32] used laser Doppler anemometry (LDA) for steady inlet flow at mid- and peak systolic flow rates to first describe the flow. The typical triple jet formation was described with two lateral orifice jets on either side of the leaflets, and a central jet between the leaflets [31]. Three-dimensionality of the flow was confirmed by Hanle et al. [14] as evidence of a four-cell streamwise vortex system in the proximal aorta was observed for steady inlet flow.

Two-component PIV (2C-PIV) has been useful in validating numerical simulations $[5,6,13]$, while providing spatial characterization of the flow. Dasi et al. [5] used 2C-PIV with pulsatile inlet conditions to describe BiMHV flow during systolic acceleration and deceleration. Ge et al. [12] analyzed data from [5] and found that cycle-to-cycle variations largely contaminated Reynolds stress calculations when ensemble averaging was used. Liu et al. [21] noted similar cycle-to-cycle variations from $2 \mathrm{C}$-LDA data and hypothesized that turbulence in the sinus may be affected by energy supplied from the previous cycle. Li et al. [20] used 2CPIV data together with a large eddy simulation model to obtain estimates of turbulent energy dissipation and length scales.

Marassi et al. [22] first used stereoscopic particle image velocimetry (SPIV) to study flow behind a bioprosthetic valve. More recently, measurements in BiMHV flow have been obtained by Kaminsky et al. $[16,17]$ using high-speed SPIV, though data was limited to a few pulsatile flow cycles due to data storage limitations. Examination of the sinus cross sectional planes in [17] showed secondary flow structures. In [16] the out-of-plane velocity was seen to reach elevated values near the sinus regions, and highlighted the importance of resolving the out-of-plane velocity component.

Unless single sample errors are extremely low, high statistical error in Reynolds stresses can result from using small datasets in turbulent flow [27]. This is discussed further in Section 3.1. To accurately record turbulent quantities in BiMHV flow, we therefore wished to obtain a large sample size SPIV dataset. To accomplish this we used an idealized valve and aortic root geometry and restricted the study to steady inlet flow. These idealized conditions limit the clinical relevance of the study, but are very well suited for CFD validation purposes. 


\section{Methods}

\subsection{Flow Loop and Test Section}

A recirculating flow loop was constructed for the experiment, as described fully in [15]. A centrifugal pump provided steady flow to an axisymmetric flow conditioning chamber upstream of the test section. The chamber housed a honeycomb flow straightener and two turbulence reduction screens. An axisymmetric contraction was attached downstream of the chamber and used a $5^{\text {th }}$ degree polynomial profile to minimize boundary layer separation at the contraction exit [2]. Downstream of the contraction, inflow piping of length $400 \mathrm{~mm}$ preceded the test section.

The use of steady flow and flow conditioning devices upstream of the test section provided controlled and welldefined inlet flow to the BiMHV, necessary for CFD validation.

Specifically, this arrangement eliminated cycle-to-cycle variations that are problematic in evaluating pulsatile flow. The inlet flow, however, differed from the in-vivo condition where pulsatile flow enters the aorta from the left ventricle. Furthermore, complex vortex development during systolic acceleration was not reflected in the current study [5].

Downstream of the contraction, inflow piping of length $400 \mathrm{~mm}$ preceded the test section. Downstream of the test section, outflow piping of length $270 \mathrm{~mm}$ ran to a flow control valve and air separator prior to entering a filling tank. The air separator was necessary to eliminate recirculating air bubbles that interfered with PIV measurements. A turbine impeller flowmeter was used for flow rate monitoring.
An axisymmetric aortic sinus design was adopted for this study. Similar axisymmetric designs have been used in previous studies and have been shown to reproduce primary flow structures of interest such as strong jet shear layers, and sinus flow recirculation relevant in the clinical setting $[5,9,10]$. The design, however, does not account for flow complexities associated with flow in three sinuses of Valsalva, or distensible aortic walls. Because of the controlled inlet flow conditions and axisymmetric aortic sinus design, the flow downstream of the BiMHV was expected to be more symmetric than the physiological case at a peak systolic Reynolds number [1]. Compared to the current design, it could also be argued that more physiological (enlarged) sinus dimensions would produce larger scale recirculation flow similar to that shown in [6].

The test section shown in Fig. 1 was similar to that used by [9] and consisted of two sections machined from cast acrylic, which enabled BiMHV insertion and removal during the PIV image calibration process. The exterior surfaces of the test section were flat to minimize optical distortions. The axisymmetric aortic sinus dimensions are shown in Fig. 1 where inlet piping of diameter $26.8 \mathrm{~mm}$ was located upstream of the valve. Downstream of the valve the sinus diameter, $d_{s}=32.0 \mathrm{~mm}$ contracted to the aortic diameter, $d=26.8$ $\mathrm{mm}$. To eliminate reflections and laser sheet blockage during the experiments, a transparent model of a Carbomedics No. 25 aortic BiMHV was used. In this study, the leaflets were fixed to eliminate fluidstructure interaction effects and also facilitate CFD validation studies with stationary meshes. The valve ring was machined from cast acrylic, and polycarbonate leaflets were fixed in the 
fully opened position. Hinge insets were not incorporated into the valve model. Detailed valve drawings are given in [15].

For the PIV experiments, the fluid used was a $61 \% \mathrm{w} / \mathrm{w}$ aqueous sodium iodide, $\mathrm{NaI}(\mathrm{aq})$, solution that matched the index of refraction, $n=1.49$, of the acrylic test section. The flow rate was adjusted and kept steady for every $1^{\circ} \mathrm{C}$ increase in fluid temperature caused by pump heat. This maintained a constant Reynolds number to preserve dynamic similarity because a blood analog was not used. The Reynolds number was 7600 , roughly corresponding to peak systole in a human aorta. For the current geometry the Reynolds number was defined as

$$
\operatorname{Re}=\frac{U_{0} d}{v}=\frac{4 Q}{\pi d v}
$$

where $U_{0}$ was the bulk inlet velocity based on the steady inflow rate $Q, d$ was the aortic diameter, and $v$ was the fluid kinematic viscosity. $U_{0}$, was calculated for each sample and used to normalize the recorded velocities. The sample average of $U_{0}$ was $0.41 \mathrm{~m} / \mathrm{s}$, and the sample average density and kinematic viscosity were $1796 \mathrm{~kg} / \mathrm{m}^{3}$ and $1.57 \times 10^{-6} \mathrm{~m}^{2} / \mathrm{s}$ respectively.

The flow was seeded with silver coated hollow glass spheres (Potters Industries, Malvern, PA) having density $1650 \mathrm{~kg} / \mathrm{m}^{3}$ and diameter $15 \mu \mathrm{m}$, ensuring that the particles responded to the flow with high fidelity [15]. Flow visualization experiments were also performed at a range of Reynolds numbers, and the images are found in [15].

\subsection{SPIV System}

Measurements were performed with an inhouse SPIV system (Fig. 1) that used a New Wave SOLO PIV 120 dual head Qswitched Nd:YAG laser emitting $532 \mathrm{~nm}$ wavelength light. The laser sheet was approximately $1 \mathrm{~mm}$ thick, and images were acquired by two 1008 x 1018 pixel Pulnix TM-1010 cameras (camera 0 and camera 1 in Fig. 1) at a sampling rate of 3 Hz. The cameras were positioned on either side of the laser sheet such that the lens planes and laser sheet plane intersected at an angle of $\alpha=30^{\circ}$. The camera planes were aligned with Scheimpflug optics at an angle of $\phi=5^{\circ}$, and data were reconstructed by positioning the cameras for angular displacement [23]. Computar TEC-M55 telecentric lenses were used to ensure paraxial recording. Video Savant 3.0 was used for image acquisition. The setup used for 2C-PIV inlet conditions measurements was the same as for SPIV, except a single camera was used, where the lens plane was positioned parallel to the laser sheet plane. Three PIV datasets were obtained:

1. Inlet Conditions Dataset (2C-PIV)

2. Full Aortic Sinus Dataset (SPIV)

3. Near-Wall Dataset (SPIV)

The measurement locations and the coordinate system used for the three datasets are shown in Fig. 1. For all three datasets, the laser sheet was positioned at the $X Y$ plane approximately on the piping centerline, perpendicular to the leaflet pivot axes. The measurement system origin was located at the leaflet tips on the aortic centerline.

Laser pulse time separation was optimized to take advantage of the system dynamic range of \pm 16 pixels for all three datasets obtained [15]. The laser pulse time separation for the SPIV measurements in the Full Aortic Sinus Dataset was $400 \mu$ s. The measurement area spanned the entire length and width of the aortic sinus, specifically, $0.05<X / d<0.80$, and -0.57 $<Y / d<0.57$ (see Fig. 1). A total of $N=$ 1105 samples were obtained for the Full Aortic Sinus Dataset. 
The purpose of the Near-Wall Dataset was to record low velocities near the sinus walls with higher accuracy. This took advantage of the full system dynamic range, but restricted the usable measurement area to only the upper and lower sinus regions. The laser pulse time separation was therefore doubled to 800 $\mu$ s. $N=718$ samples were collected. The Near-Wall Dataset extended from $0.05<$ $X / d<0.50$. The measurement region in the upper sinus spanned from $0.45<Y / d<$ 0.57 , and for the lower sinus region it spanned from $-0.57<Y / d<-0.45$ as shown in Fig. 1.

The Inlet Conditions Dataset recorded the inlet flow conditions and $N=1080$ samples were obtained with a laser pulse time separation of $400 \mu \mathrm{s}$. The measurement location for the Inlet Conditions Dataset extended from -3.34< $X / d<-2.95$, and spanned $-0.48<Y / d<$ 0.48 as shown in Fig. 1.

\subsection{Image Calibration}

In SPIV, two cameras record distorted mirror images of the same plane, which requires that the images be projected onto a common plane prior to calculating particle displacements. The SPIV calibration method employed image dewarping which mapped pixel locations to their back-projected locations. The SPIV images exhibited two types of image distortion:

1. Projective distortion from Scheimpflug angle tilt of the CCD image plane

2. Non-linear distortions from viewing the measurement plane through the curved aortic sinus (see Fig. 1)

During image calibration, a calibration grid with $1 \times 1 \mathrm{~mm}$ uniformly spaced grid nodes was placed in the test section and imaged. The resulting image of distorted grid node locations was dewarped back to uniform grid node locations.

Two different dewarping algorithms were tested and their errors compared. Error analysis between a conventional $2^{\text {nd }}$ order polynomial method and an unconventional local weighted mean (LWM) method is discussed extensively in [15]. It was found that the non-linear image distortions present in the images resulted in unacceptable errors (maximum 5.5 pixels, average 0.9 pixels) using the $2^{\text {nd }}$ order polynomial transformation. However, the low dewarping error using the LWM method was acceptable (maximum 0.8 pixels, average 0.1 pixels) for accurate SPIV measurements (where 1 pixel $=33$ $\mu \mathrm{m})$. This is the first time that a LWM dewarping method has been used in SPIV work, and it is likely that SPIV studies with similar non-linear optical distortions can be dewarped in a similar way.

\section{$2.4 \quad$ Image Processing}

Particle images were processed with an adaptive cross correlation PIV algorithm [28], with a first pass $64 \times 64$ pixel interrogation area and a second pass $32 \mathrm{x}$ 32 pixel interrogation area. For the Full Aortic Sinus Dataset and Near-Wall Dataset, the spatial resolution was $\Delta X=$ $0.59 \mathrm{~mm}$ and $\Delta Y=0.53 \mathrm{~mm}$ with a $50 \%$ interrogation area overlap. Equations from [30] were used to reconstruct the 3C velocity vectors. For the Inlet Conditions Dataset the spatial resolution was $\Delta X=\Delta Y$ $=0.47 \mathrm{~mm}$ with a $50 \%$ interrogation area overlap. 


\section{$3 \quad$ Results}

Total velocities, $u, v, w$, ensemble averaged mean velocities $U, V, W$, and turbulence velocities $u^{\prime}, v^{\prime}, w^{\prime}$ corresponded to the $X, Y$ and $Z$ directions respectively. Reynolds stress components were averaged and are presented as $\left\langle u_{i}^{\prime} u_{j}^{\prime}\right\rangle$. All mean velocities have been normalized by the bulk inlet velocity, $U_{0}$, and all Reynolds stress components by $U_{0}{ }^{2}$. All distances have been normalized by aortic diameter, $d$.

\subsection{Error Analysis}

Errors for the two SPIV datasets were calculated based on uncertainty estimates in the experimental setup and on measured turbulence intensity. The errors in mean velocity and Reynolds stress are given as [29]

$$
\begin{gathered}
\sigma_{\text {mean }}=\sqrt{\frac{\sigma_{T I}^{2}+\sigma_{\text {inst }}^{2}}{N}}+e_{\text {mean-IA }} \\
\sigma_{R S}=\sqrt{\frac{2\left(\sigma_{T I}^{4}+\sigma_{\text {inst }}^{4}\right)}{N}}+e_{R S-I A}
\end{gathered}
$$

where $\sigma_{\text {mean }}$ is error in mean velocity, $\sigma_{\text {inst }}$ is error in a single sample, and $\sigma_{T I}$ is the turbulence intensity pertaining to each of the $u, v$ and $w$ velocities. The variable $\sigma_{R S}$ is error in Reynolds stress for each of the six stress components. This analysis pertains to homogeneous turbulence, which was most closely represented in the lateral orifice jet cores where an average of $\sigma_{T I-u}=\sigma_{T I-v}=20 \%$ was recorded for $u$ and $v$ component velocities. For the current study, additional systematic errors were attributed to interrogation area overlap, $e_{\text {mean-IA }}$ and $e_{R S-I A}[15]$. Values of $e_{\text {mean-IA }}=$ $3 \%$ and $e_{R S-I A}=6 \%$ were found for the inplane mean velocities ( $U$ and $V$ ) and
Reynolds stresses $\left\langle u^{\prime} u^{\prime}\right\rangle,\left\langle v^{\prime} v^{\prime}\right\rangle,\left\langle u v^{\prime}\right\rangle$ respectively. Values of

$e_{\text {mean-IA-W }}=5 \%$ and $e_{R S-I A-w^{\prime}}=10 \%$ were found for the out-of-plane velocity, $W$, and Reynolds stresses $\left\langle w^{\prime} w^{\prime}\right\rangle,\left\langle u w^{\prime}\right\rangle$, $<v^{\prime} w^{\prime}>$ respectively [15].

Errors with respect to correlation peak locking and signal-to-noise ratio were minimized. In addition, image registration, camera angle, and crosscorrelation algorithm accuracy were accounted in the error calculation. Errors in a single sample were determined to be $\sigma_{\text {inst }-u}=\sigma_{\text {inst }-v}=13 \%$ for the in-plane component velocities. In general, single sample errors $\sigma_{\text {inst-w }}$ in $w$ were expected to be at least a factor of $1 / \tan (\alpha)=1.73$ larger than that of $u$ and $v$ [23]. With additional errors in image registration affecting $w$, a value of $\sigma_{\text {inst-w }}=24 \%$ was calculated [15].

Flow unsteadiness and thus symmetry breaking (see Section 3.2) about the $X Y$ plane contributed to an overestimation of out-of-plane turbulence intensity. This was similar to the problem encountered when making turbulence measurements in a flapping shear layer. The effect was further exaggerated due to higher error of the out-of-plane component and low magnitude in $W$, and was most prevalent in regions external to the lateral orifice jets, at roughly $|Y / d|>0.40$. Thus for Fig. 7, the plots of Reynolds stresses containing $w$, only data for $-0.40<Y / d<0.40$ were considered valid. To estimate the error in quantities containing out-of-plane data, the out-of-plane turbulence intensity was assumed equal to that of the in-plane values, thus a value of $\sigma_{T I-w}=20 \%$ was used.

Using these values with sample size $N$ for the datasets, the overall error estimates are given in Table 1. Spatial variation of turbulence intensity meant that error was 
also dependant on measurement location. Values in Table 1 are therefore considered only to be an approximation of the error.

Lastly, relative to the rest of the velocity field, velocities for $X / d>0.60$ had slightly higher errors due to a small amount of image blurring from viewing through the aortic sinus contraction region (see Fig. 1). This effect was noticeable in out-of-plane velocity and therefore Fig. 5 and Fig. 7, span only $0.05<X / d<0.60$, instead of $0.05<X / d<0.80$ as shown in figures giving in-plane velocities.

\subsection{Inlet Conditions}

The average value of $U / U_{0}$ across the inlet cross-section was expected to be approximately unity, which was confirmed by calculation of $U_{0}$ from flow meter readings. Specifically, an average value of $U / U_{0}=1.03$ was found for all inlet condition axial locations. The 3\% higher measured value was within uncertainty estimates (see Table 1).

Fig. 2 shows mean axial velocity, $U / U_{0}$, and axial rms velocity, $\left\langle u^{\prime} u^{\prime}\right\rangle^{1 / 2} / U_{0}$, profiles at three axial locations. In Fig. 2, a typical turbulent pipe flow profile was observed, and profiles at all axial locations almost completely overlapped indicating that the inlet flow could be considered fully developed. A small asymmetry in mean velocity about the pipe centerline $(X$ axis) can be seen in Fig. 2. Profile asymmetry was quantified as the percent difference between velocity vectors at the same distance from each wall. The majority of the profile, for $-0.34<Y / d<$ 0.34 , was symmetric with a less than $3 \%$ velocity difference, which was within measurement accuracy (see Table 1). However, from $0.34<|Y / d|<0.45$, the asymmetry increased from $5 \%$ at $Y / d=$
0.34 to $17 \%$ at $Y / d=0.45$. Within this region, velocity on the lower piping side $(Y<0)$ was higher than on the upper piping side $(Y>0)$. Inlet turbulence had a peak value of $\left\langle u^{\prime} u^{\prime}\right\rangle^{1 / 2} / U_{0}$ on the lower piping side $(Y<0) 33 \%$ higher than that on the upper side $(Y>0)$. We hypothesize that the asymmetry in mean velocity near the pipe walls was due to uneven particle coating of the inlet piping walls which resulted from running a large number of experiments with the flow loop. The differing surface roughness on one side of the piping compared to the other likely caused the asymmetry in mean velocity and thus led to the asymmetry in inlet turbulence.

\subsection{SPIV Results}

\section{General Flow Characteristics}

Contour plots of the ensemble-averaged velocity field of the Full Aortic Sinus Dataset for $U / U_{0}, V / U_{0}$, and $W / U_{0}$ are shown in Fig. 3a, b, and c respectively. In all figures, flow is from left to right, and the leaflet tips are shown as boxes on the $Y$-axis at $Y / d= \pm 0.14$. Contour levels have been adjusted to be within uncertainty estimates.

As expected, axial velocity in the lateral orifice jets (at roughly $0.20<|Y / d|<0.40$ ) was clearly dominant in the flow. The highest axial velocities in the flow were recorded in the lateral orifice jets of roughly $U / U_{0}=2.60$ for $X / d<0.21$. The central orifice jet (at $-0.14<Y / d<0.14$ ) had a peak velocity of roughly $U / U_{0}=$ 2.20 for $X / d<0.21$. In Fig. 3a, regions of high shear are indicated by high $\partial\left(U / U_{0}\right) /$ $\partial Y$. This was the dominant gradient of the flow, and peak gradient values occurred near the upper and lower lateral orifice jet shear layers around $0.40<|Y / d|<0.45$. The lateral orifice jet shear layers were 
thus sites of intense mixing, which has also been noted by $[19,33]$ and others. The plot of $V / U_{0}$, in Fig. 3b shows that the flow was relatively symmetric about the $X$ axis. The out-of-plane velocity, $W / U_{0}$, (shown in Fig. 3c) and transverse velocity, $V / U_{0}$, were of similar magnitude indicating that the out-of-plane component contributed significantly to the flow, which has been also observed by Kaminsky et al. [16]. The Near-Wall Dataset had good resolution of the low velocities observed near the sinus walls (Fig. 4). In the upper sinus region (Fig. 4a), typical flow reversal and well-defined recirculation was apparent. The type of flow was similar to that seen in flow over a backward facing step, as flow separation occurred at the step edge (in this case, the valve ring at $X / d=-0.16$ and $Y / d= \pm 0.38$ ). A similar flow pattern was seen in the lower sinus region (Fig. 4b) but the recirculation bubble differed slightly in both size and shape compared to that in the upper sinus region. A relatively small asymmetry in inlet mean velocity and inlet turbulence was observed in the current study (see Section 3.1), and thus likely affected the upper and lower sinus region flow [19]. Tests were performed with varied valve position to check for valve geometry effects. The tests showed no noticeable change in flow structure; however, a valve geometrical imperfection such as a small error in leaflet angle or leaflet trailing edge profile could not be fully ruled out as a contributing factor to the small sinus region asymmetry.

\section{D Flow Structure}

Fig. 5a shows a contour plot of $W / U_{0}$ exhibiting the three dimensional nature of the flow also noted in other experiments $[1,14,16]$. Contour levels correspond to error estimates and thus Fig. 5a shows significant out-of-plane motion.
Discussion of possible 3D structures in the flow is found in Section 4.

In a low Reynolds number CFD simulation, Ge et al. [10] found symmetry breaking about the $X Y$ plane at $\mathrm{Re}=750$. It is therefore likely that there was some degree of asymmetry about the $X Y$ plane in the current study (where $\mathrm{Re}=7600$ ). Given that the measurement plane was aligned with the $X Y$ plane, even a slight flow asymmetry about this plane would cause a net flow through the measurement plane. Fig. 5a shows that a net throughplane flow was present as larger regions of positive $W / U_{0}$ velocity can be seen relative to regions of negative $W / U_{0}$.

\section{Reynolds Stresses}

Due to the idealized conditions used in the present study, applicability to in-vivo blood element damage based on Reynolds stress results is limited. Furthermore, Reynolds stresses have been shown to be only one factor in many, including eddy decay time and viscous stresses, that affect element damage $[7,12]$. The Reynolds stress profiles are however useful references in CFD validation and aid in the study of BiMHV jet structure and shear layer mixing.

Fig. 6 shows the average Reynolds stresses, $<u^{\prime} u^{\prime}>/ U_{0}^{2},<v^{\prime} v^{\prime}>/ U_{0}^{2}$, $<u^{\prime} v^{\prime}>/ U_{0}^{2}$, and Fig. 7 shows average Reynolds stresses, $\left\langle w^{\prime} w^{\prime}>/ U_{0}{ }^{2}\right.$, $<u^{\prime} w^{\prime}>/ U_{0}^{2},<v^{\prime} w^{\prime}>/ U_{0}^{2}$. For the first time, average Reynolds stress fields are shown for all six components. In the figures, apparent discontinuities between neighboring contour levels were due only to contour level choice, based on error estimates. Because of the relatively high error estimates of average Reynolds stress (see Table 1), this discussion focuses on qualitative interpretation.

Relatively low Reynolds stress was seen in the lateral orifice jets (at roughly $0.20<$ 
$|Y / d|<0.40)$, in contrast to the higher magnitudes in the central jet (roughly $-0.14<Y / d<0.14)$. In the central jet, regions of elevated Reynolds normal stress (Fig. 6a and b, and less pronounced in Fig $7 \mathrm{c})$ are shown on either side of the $X$ - axis. These off-axis peaks are commonly observed in free jets [25], which demonstrate a somewhat surprising similarity between the central jet and a free jet given the presence of leaflets and the effect of confinement. Central jet selfsimilarity and a decay rate similar to a free rectangular jet have also been observed [18] and is discussed further in [15].

Fig. 7 shows plots of the Reynolds stresses containing the out-of-plane turbulence velocity, $\left(\left\langle u^{\prime} w^{\prime}\right\rangle / U_{0}^{2},\left\langle v^{\prime} w^{\prime}\right\rangle / U_{0}^{2}\right.$ and $\left.\left\langle w^{\prime} w^{\prime}\right\rangle / U_{0}^{2}\right)$, and illustrates high

Reynolds stresses in the central jet region as well. Also, off-axis peaks in Reynolds shear stress were seen in the leaflet wakes (roughly $Y / d= \pm 0.10$ ) which are most apparent in the plots of $\left\langle u^{\prime} v^{\prime}\right\rangle / U_{0}^{2}$ and $\left\langle v^{\prime} w^{\prime}\right\rangle / U_{0}^{2}$. This type of behavior has also been observed previously in confined multiple jets where similar wakes were present [8].

In regions of high mean shear such as the lateral orifice jet shear layers, increased Reynolds stresses were expected [25], and indeed high values of $\left\langle u^{\prime} u^{\prime}\right\rangle / U_{0}^{2}$, $\left\langle v^{\prime} v^{\prime}\right\rangle / U_{0}^{2}$, and $\left\langle u^{\prime} v^{\prime}\right\rangle / U_{0}^{2}$ are seen in Fig. 6 for $|Y / d|>0.4$. Because of error considerations (see Section 3.1), the regions exterior to the lateral orifice jet shear layers $(|Y / d|>0.4)$ are not shown for plots of $\left\langle w^{\prime} w^{\prime}\right\rangle / U_{0}^{2},<u$ ' $\left.w^{\prime}\right\rangle / U_{0}^{2}$ and $\left\langle v^{\prime} w^{\prime}\right\rangle / U_{0}^{2}$ in Fig. 7, though a similar behavior was expected.

\section{Discussion}

In previous BiMHV studies, three dimensionality of the flow has been observed. Kaminsky et al. [16] found significant out-of-plane motion in the sinus regions and leaflet wakes during systole acceleration. Similarly, the two lobed regions of elevated $W / U_{0}$ (see dashed box region in Fig. 5a) resulted from leaflet wake disturbance. In addition, 3D structures have been identified in BiMHV flow with steady inflow conditions by Hanle et al. [14]. They found evidence of four large streamwise vortices in the proximal aorta (just downstream of the aortic sinus), two above and two below the $X Y$ plane, as shown in Fig. 5c. In the current study, the finite measurement plane thickness and likelihood of symmetry breaking about the $X Y$ plane enabled the visualization of similar structures. Examination of the contour plot in Fig. 5a shows evidence of counterrotating streamwise (i.e. $\Omega_{x}$ ) vortices that extended longitudinally along the aortic sinus. Positive $W / U_{0}$ velocity occurred near the sinus walls at $Y / d= \pm 0.60$ which appeared to correspond to the regions of negative $W / U_{0}$ velocity closer to the aortic centerline. Although data from measurement planes above and below the $X Y$ plane are needed for confirmation, it was possible that the vortices comprised a four-cell streamwise vortex structure in the aortic sinus as shown in Fig. 5 b.

It is unclear if similar 3D structures are formed in-vivo, where pulsatile inlet flow and three sinuses of Valsalva are present. Therefore we feel it is not appropriate to comment on the clinical relevance of the four-cell streamwise vortex pattern until further, more physiological data is collected.

In regards to the plots of Reynolds stress in Fig. 6 and 7, the data suggests that downstream of the valve, the central jet, lateral orifice jet shear layers, and leaflet wakes were the most turbulent regions in 
the flow, which is in agreement with [1,21,32 and others]. In the current study, obtaining turbulence data for the out-ofplane velocity using SPIV enabled spatial visualization of all six Reynolds stress components, information that had previously been unavailable.

BiMHV flow was seen to be sensitive to inlet conditions, as a relatively small asymmetry in inlet mean velocity and inlet turbulence likely caused differing flow patterns in the sinus regions. This emphasizes the need for well defined inlet conditions in BiMHV studies, especially when CFD validation is required.

The above illustrates the highly complex nature of BiMHV flow as it combines the unsteady flow of three confined jets, leaflet wakes, and sinus recirculation regions. Because of the idealized aortic geometry, fixed valve leaflets, absence of hinge insets, and steady flow regime used in the current study, one should not make direct comparison to in-vivo hemodynamics from the current discussion. The data does, however, provide a basis for future experimental studies using SPIV. Using high-speed SPIV with large data storage capability, one could collect enough samples for mean flow and Reynolds stress mapping throughout the full cardiac cycle $[5,16]$.

Due to the 3D nature of BiMHV flow it is recommended that future experimental studies use SPIV. In addition, the 3D data currently available from advanced CFD simulations reinforces the need for validation datasets with three velocity components. The Full Aortic Sinus Dataset is posted (https://tspace.library.utoronto.ca/) and is well-suited for initial steady inflow validation as it has well defined inlet conditions and a relatively low error in mean velocity. The dataset can be used as a starting point for CFD validation with a steady flowrate prior to pulsatile flow simulation. It consists of 1982 velocity vectors in 1105 realizations. Instantaneous and mean velocities, and average Reynolds stress values are given.

\section{Acknowledgement}

This work was supported by NSERC (C.R.E., P.S.). C.H. was supported by NSERC PGS-M funding. In addition, the Canada Research Chairs Program (C.R.E.) is gratefully acknowledged. 


\section{References}

1) Balducci, A., Grigioni, M., Querzoli, G., Romano, G. P., Daniele, C., D'Avenio, G., et al., 2004, "Investigation of the Flow Field Downstream of an Artificial Heart Valve by Means of PIV and PTV". Experiments in Fluids, 36(1), pp. 204-213.

2) Bell, J. H., \& Mehta, R. D., 1988, "Contraction Design for Small Low-Speed Wind Tunnels. Technical Report No. 177488, NASA.

3) Borazjani, I., Ge L., Sotiropoulos F., 2010, "High-Resolution Fluid Structure Interaction Simulations of Flow Through a Bileaflet Mechanical Heart Valve in an Anatomic Aorta". Annals of Biomedical Engineering, 38(2), pp. 326-344.

4) Brucker, C., 1997, "Dual-camera DPIV for Flow Studies Past Artificial Heart Valves". Experiments in Fluids, 22(6), pp. 496-506.

5) Dasi, L. P., Ge, L., Simon, H. A., Sotiropoulos, F., \& Yoganathan, P. A., 2007, "Vorticity Dynamics of a Bileaflet Mechanical Heart Valve in an Axisymmetric Aorta”. Physics of Fluids, 19(6).

6) De Tullio, M. D., Cristallo, A., Balaras, E., Verzicco, R., 2009, “Direct Numerical Simulation of the Pulsatile Flow Through an Aortic Bileaflet Mechanical Heart Valve". Journal of Fluid Mechanics, 622, pp. 259-290.

7) Dooley, P., Quinlan, N., 2009, "Effect of Eddy Length on Mechanical Loading of Blood Cells in Turbulent Flow". Annals of Biomedical Engineering, 37(12), pp. 2449-2458.

8) Fabris, G., \& Fejer, A. A., 1974, "Confined Mixing of Multiple Jets". Transactions of the ASME, 96, Series 2(2).

9) Fontaine, A. A., Ellis, J. T., Healy, T. M., Hopmeyer, J., \& Yoganathan, A. P., 1996, "Identification of Peak Stresses in Cardiac Prostheses: A Comparison of Twodimensional Versus Three-dimensional Principal Stress Analyses". ASAIO Journal, 42(3), pp. 154-163.

10) Ge, L., Jones, S. C., Sotiropoulos, F., Healy, T. M., \& Yoganathan, A. P., 2003, "Numerical Simulation of Flow in Mechanical Heart Valves: Grid Resolution and the Assumption of Flow Symmetry. Journal of Biomechanical Engineering, 125(5), pp. 709-718.

11) Ge, L., Leo, H., Sotiropoulos, F., \& Yoganathan, A. P., 2005, "Flow in a
Mechanical Bileaflet Heart Valve at Laminar and Near-Peak Systole Flow Rates: CFD Simulations and Experiments". Journal of Biomechanical Engineering, 127(5), pp. 782-797.

12) Ge, L., Dasi, L. P., Sotiropoulos, F., \& Yoganathan, A. P., 2008, "Characterization of Hemodynamic Forces Induced by Mechanical Heart Valves: Reynolds vs. Viscous Stresses". Annals of Biomedical Engineering, 36(2), pp. 276-297.

13) Guivier-Curien C., Deplano V., Bertrand E., "Validation of a Numerical 3D FluidStructure Interaction model for a Prosthetic Valve Based on Experimental PIV Measurements". Medical Engineering and Physics, 31, pp. 986-993.

14) \Hanle, D. D., Harrison, E. C., Yoganathan, A. P., \& Corcoran, W. H., 1988, "In Vitro Fluid Dynamics of the St. Jude Valve Prosthesis in Steady and Pulsatile Flow". Engineering in Medicine, 17(4), pp. 181-187.

15) Hutchison, C., 2009, "Stereoscopic PIV in Steady Flow Through a Bileaflet Mechanical Heart Valve". M.A.Sc thesis, University of Toronto.

16) Kaminsky, R., Kallweit, S., Weber, H. -., Claessens, T., Jozwik, K., \& Verdonck, P., 2007, "Flow Visualization Through Two Types of Aortic Prosthetic Heart Valves Using Stereoscopic High-speed Particle Image Velocimetry". Artificial Organs, 31(12), pp. 869-879.

17) Kaminsky, R., Kallweit, S., Rossi, M., Morbiducci, U., Scalise, L., Verdonck, P., et al., 2008, "PIV measurements of flows in artificial heart valves". Particle Image Velocimetry: New Developments and Recent Applications, Schroeder, A., \& Willert, C., eds., Springer-Verlag, Berlin.

18) Krothapalli, A., Baganoff, D., \& Karamcheti, K., 1981, "On the Mixing of a Rectangular Jet". Journal of Fluid Mechanics, 107, pp. 201-220.

19) Le, H., Moin, P., \& Kim, J., 1997, “Direct Numerical Simulation of Turbulent Flow Over a Backward-facing Step. Journal of Fluid Mechanics”, 330, pp. 349-374.

20) Li, C., Lo, C., Lu, P., 2010, "Estimation of Viscous Dissipative Stresses Induced by a Mechanical Heart Valve Using PIV Data". Annals of Biomedical Engineering, 38(3), pp. 903-916.

21) Liu, J. S., Lu, P. C. 1., \& Chu, S. H., 2000, "Turbulence Characteristics Downstream 
of Bileaflet Aortic Valve Prostheses". Journal of Biomechanical Engineering, 122(2), pp. 118-24.

22) Marassi, M., Castellini, P., Pinotti, M., \& Scalise, L., 2004. "Cardiac Valve Prosthesis Flow Performances Measured by $2 \mathrm{D}$ and 3D-Stereo Particle Image Velocimetry". Experiments in Fluids, 36(1), pp. 176-186.

23) [23] Prasad, A. K., 2000,

"Stereoscopic Particle Image

Velocimetry". Experiments in Fluids, 29(2), pp. 103-116.

24) Sotiropoulos, F., Borazjani, I., 2009, “A Review of State-of-the-Art Numerical Methods for Simulating Flow Through Mechanical Heart Valves". Medical and Biological Engineering and Computing, 47, pp. 245-256.

25) Tennekes, H. \& Lumley, J. L., 1972, A First Course in Turbulence. MIT Press, Cambridge, Mass.

26) Shahbazi, K., 2007, “A Parallel Highorder Discontinuous Galerkin Solver for the Unsteady Incompressible NavierStokes Equations in Complex Geometries". PhD thesis, University of Toronto.

27) Ullum, U., Schmidt, J. J., Larsen, P. S., \& McCluskey, D. R., 1998, "Statistical Analysis and Accuracy of PIV Data.
Journal of Visualization, 1(2), pp. 205216.

28) Usera, G., 1999, “Adaptive Algorithms for PIV Image Analyzing”. Technical report, Institute of Fluid Mechanics and Environmental Engineering (IMFIA) University of the Republic, Montevideo, Uruguay.

29) Wernet, M. P., Subramanian, A., Mu, H., \& Kadambi, J. R., 2000, "Comparison of Particle Image Velocimetry and Laser Doppler Anemometry Measurements in Turbulent Fluid Flow". Annals of Biomedical Engineering, 28(11), pp. 1393-1394.

30) Willert, C., 1997, "Stereoscopic Digital Particle Image Velocimetry for Application in Wind Tunnel Flows". Measurement Science and Technology, 8(12), pp. 1465-1479.

31) Yoganathan, A. P., Chaux, A., \& Gray, R. J., 1984, "Bileaflet, Tilting Disc and Porcine Aortic Valve Substitutes: In Vitro Hydrodynamic Characteristics". Journal of the American College of Cardiology, 3(2 I), pp. 313-320.

32) Yoganathan, A. P., Yi-Ren Woo, \& Hsing-Wen Sung, 1986, “Turbulent Shear Stress Measurements in the Vicinity of Aortic Heart Valve Prostheses". Journal of Biomechanics, 19(6), pp. 433-442. 


\begin{tabular}{|c|c|c|c|c|c|}
\hline & & \multicolumn{4}{|c|}{ Error in: } \\
\hline Dataset Name & $\mathbf{N}$ & $\mathbf{U}, \mathbf{V}$ & $\mathbf{W}$ & $\begin{array}{l}<\mathbf{u}^{\prime} \mathbf{u}^{\prime}> \\
<\mathbf{v}^{\prime} \mathbf{v}^{\prime}> \\
<\mathbf{u}^{\prime} \mathbf{v}^{\prime}>\end{array}$ & $\begin{array}{l}<\mathbf{w}^{\prime} \mathbf{w}^{\prime}> \\
<\mathbf{u}^{\prime} \mathbf{w}^{\prime}> \\
<\mathbf{v}^{\prime} \mathbf{w}^{\prime}>\end{array}$ \\
\hline Inlet Condtions & 1080 & $\pm 3 \%$ & N/A & $\pm 14 \%$ & N/A \\
\hline Full Aortic Sinus & 1105 & $\pm 4 \%$ & $\pm 6 \%$ & $\pm 24 \%$ & $\pm 40 \%$ \\
\hline Near-Wall Sinus & 718 & $\pm 4 \%$ & $\pm 7 \%$ & $\pm 29 \%$ & $\pm 47 \%$ \\
\hline
\end{tabular}

Table 1. Error estimates for the three datasets. $N$ is number of acquired velocity fields; $U, V$ and $W$ are mean velocity components; $u^{\prime}, v^{\prime}$ and $w^{\prime}$ are fluctuating components 


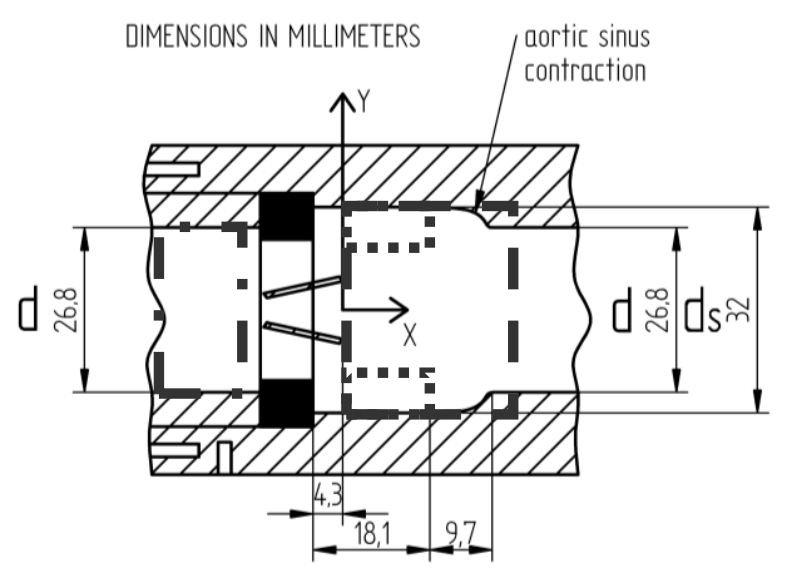

(a)

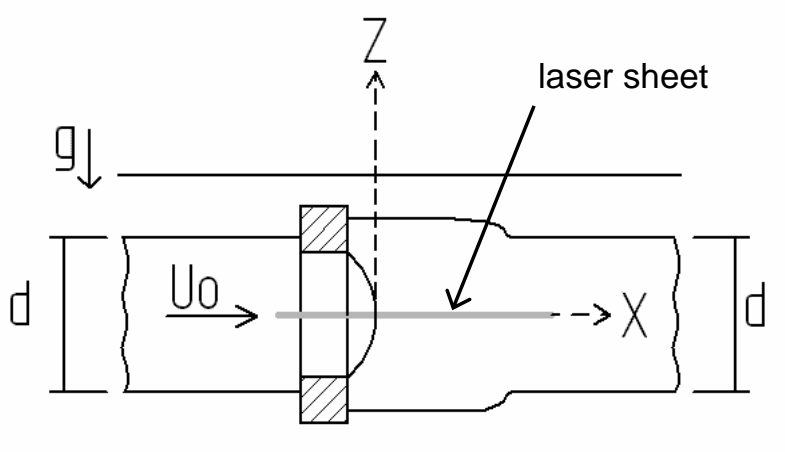

(b)

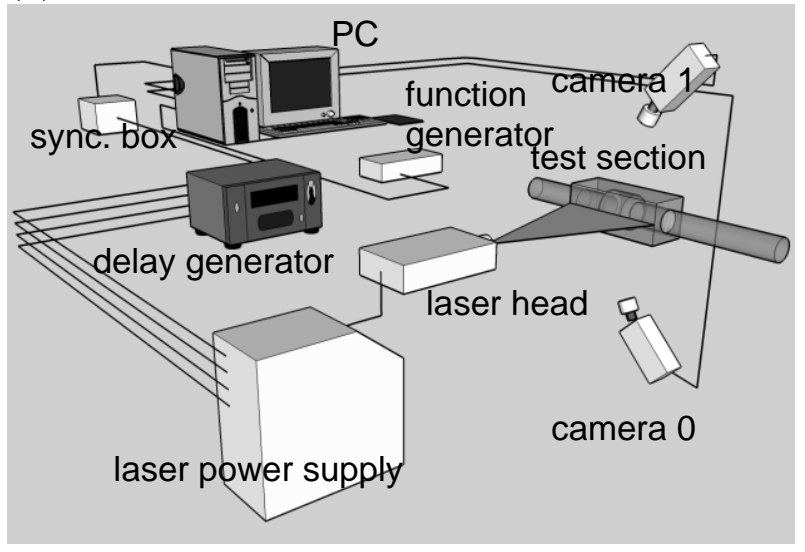

(c)

Figure 1. (a) Plan view of axisymmetric aortic sinus geometry (not to scale). Dashed lines show the measurement locations of the 2C-PIV Inlet Conditions Dataset (- . -), SPIV Full Aortic Sinus Dataset (- - -) and SPIV Near Wall Dataset (... .). (b) Side view of test section (not to scale) showing bulk inlet flow velocity $U_{0}$ and laser sheet positioning. (c) In-house SPIV setup. The PC was used to relay signals from the cameras to the delay generator which was used to trigger the laser pulses 


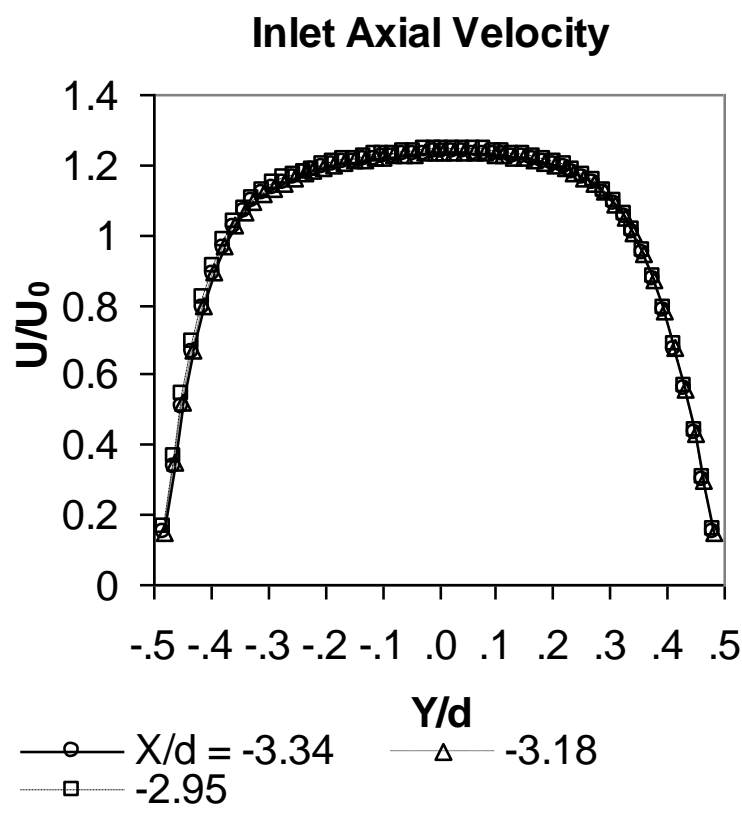

(a)

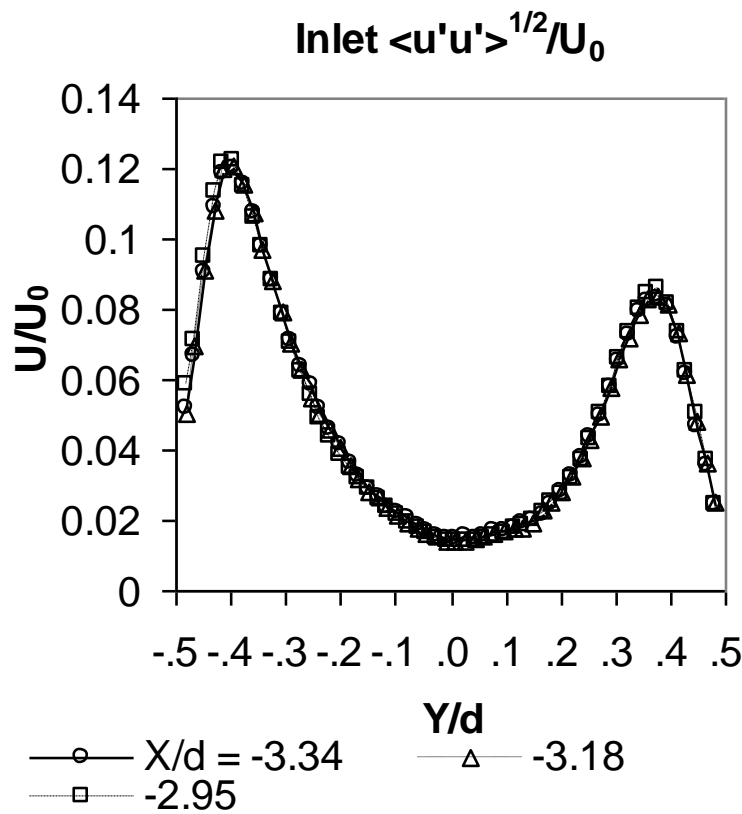

(b)

Figure 2. Mean axial velocity and axial turbulence profiles of inlet conditions. Mean profiles show a fully developed turbulent velocity profile as downstream data points almost completely overlapped, though peak turbulence was notably higher at negative compared to positive $Y$ values 

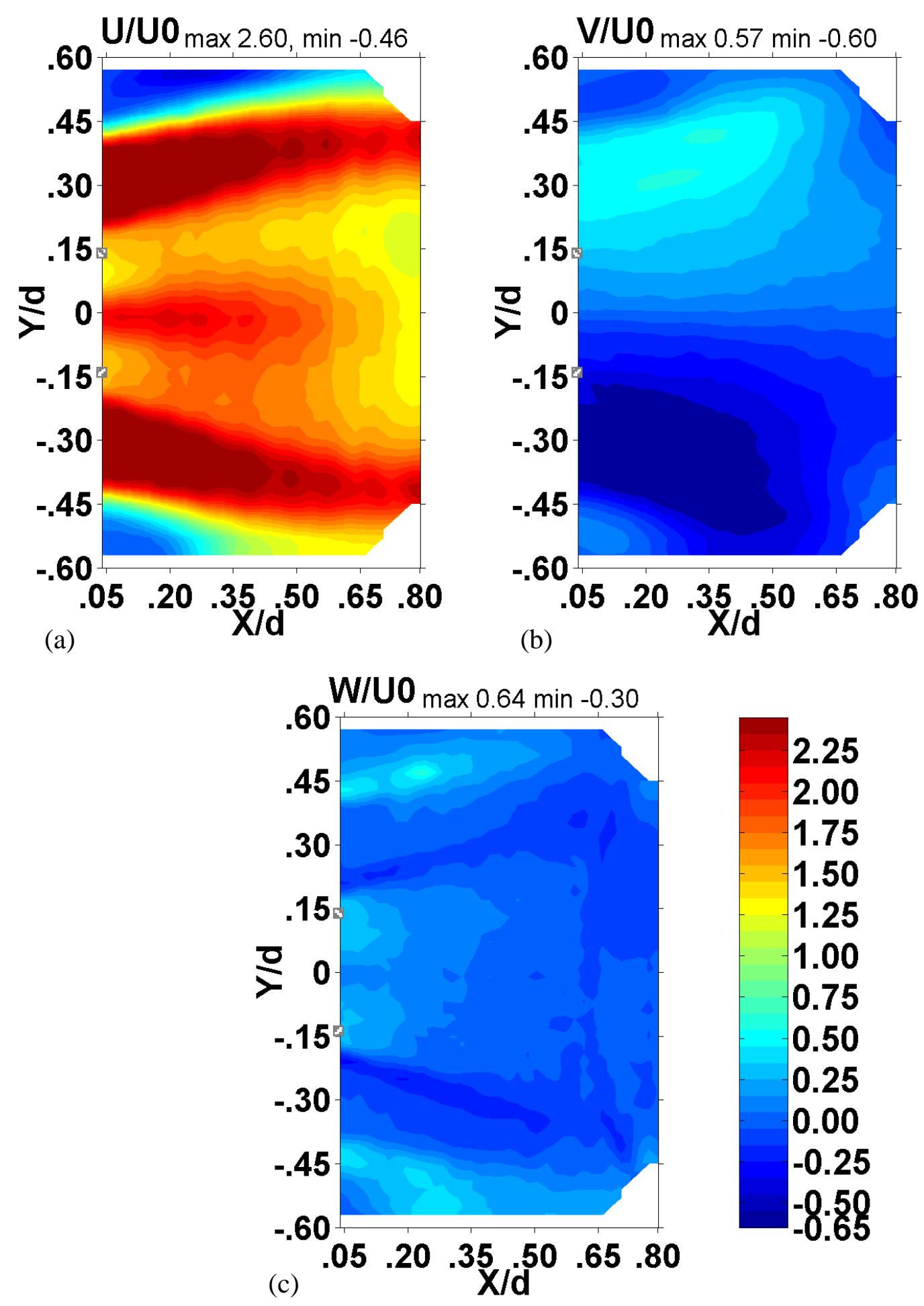

Figure 3. Mean velocity field for the Full Aortic Sinus Dataset. Leaflet tips were located at $Y / d \pm 0.14$ and $X / d=0$, and are shown as hatched boxes on the plots. $W / U_{0}$ was of similar magnitude to $V / U_{0}$ in the lateral orifice jet shear layers. Contour levels correspond to the full scale error in $W / U_{0}$ of $\pm 6 \% \times 0.64= \pm 0.04$, to show significant values of all three velocity components 


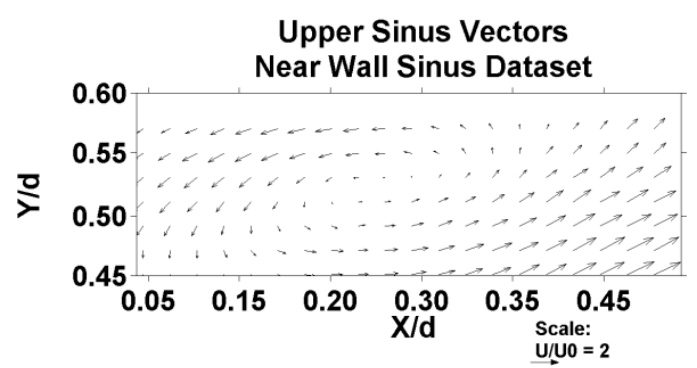

(a)

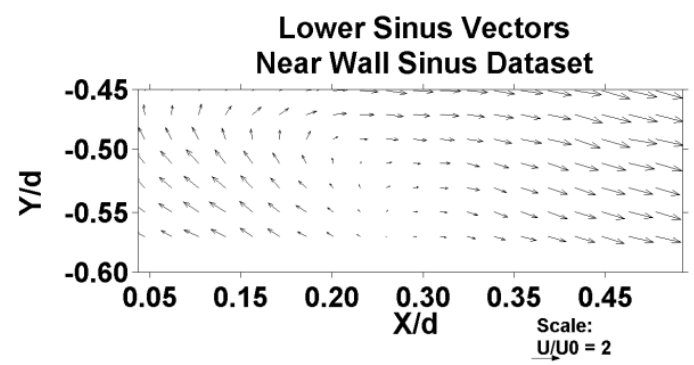

(b)

Figure 4. Upper (a) and lower (b) sinus region mean flow from the Near-Wall Dataset. An atypical recirculation was seen in the lower sinus region that may have been due to inlet turbulence. The aortic sinus wall was located at $Y / d= \pm 0.60$. 


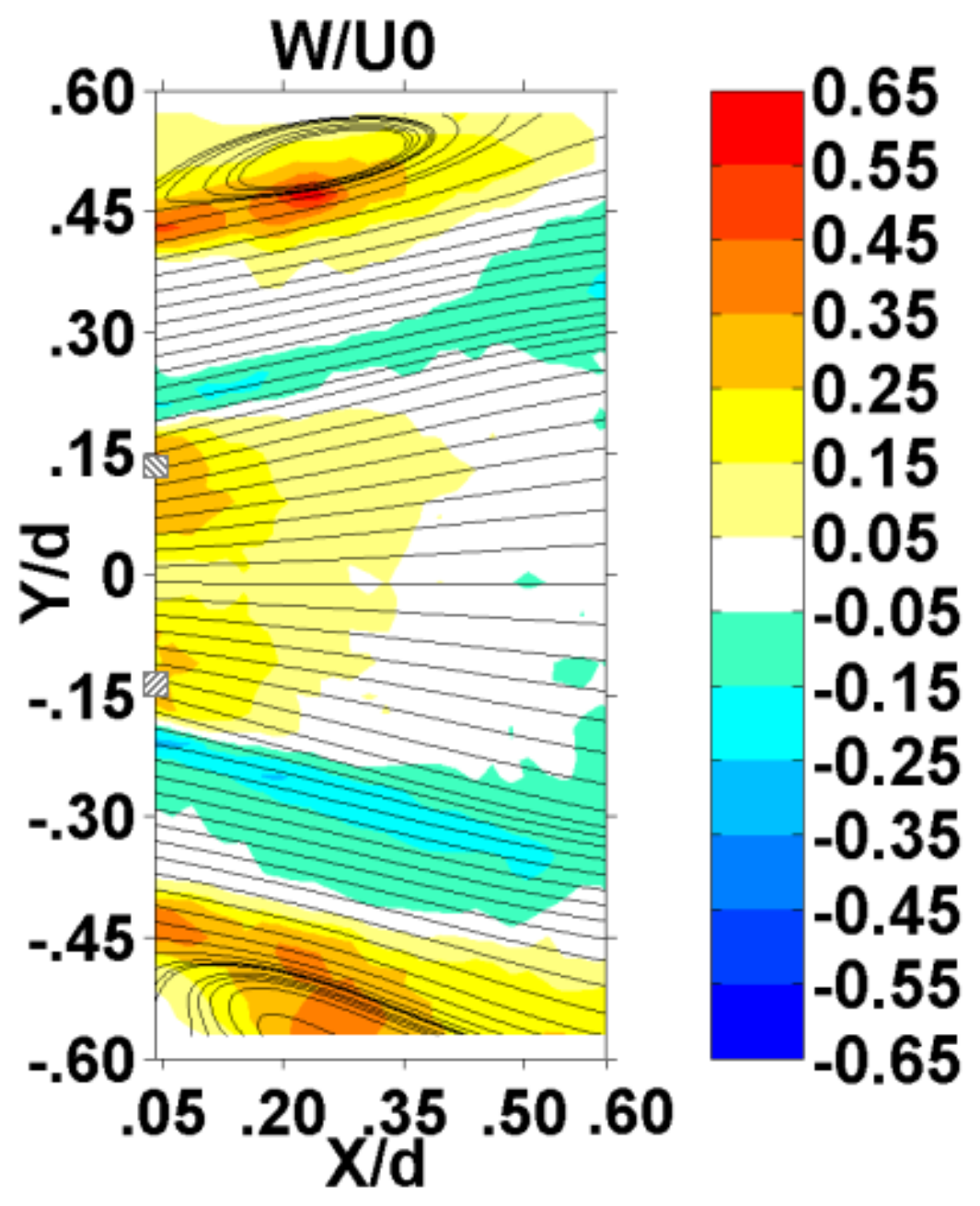

(a)

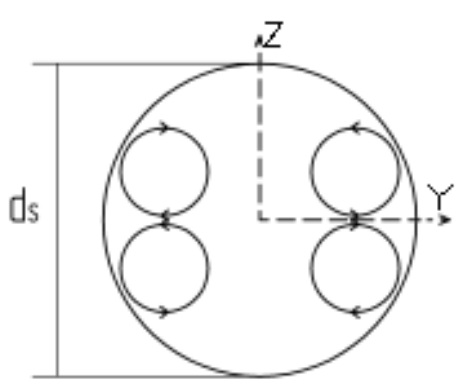

(b)

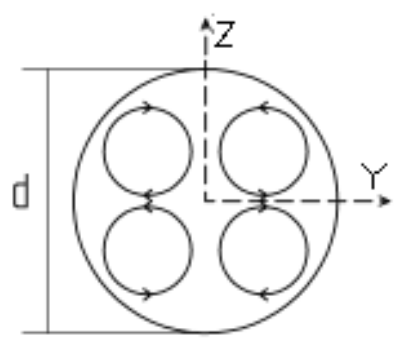

(c)

Figure 5. (a) Contour plot of $W / U_{0}$ for the Full Aortic Sinus Dataset, with streamlines computed from the $U$ and $V$ velocities overlain. Leaflet tips were located at $Y / d \pm 0.14$ and $X / d=0$, and are shown as hatched boxes. (b) Possible four-cell streamwise vortex structure in the sinus. (c) The four-cell vortex structure downstream of the sinus observed by Hanle et al. [6] 

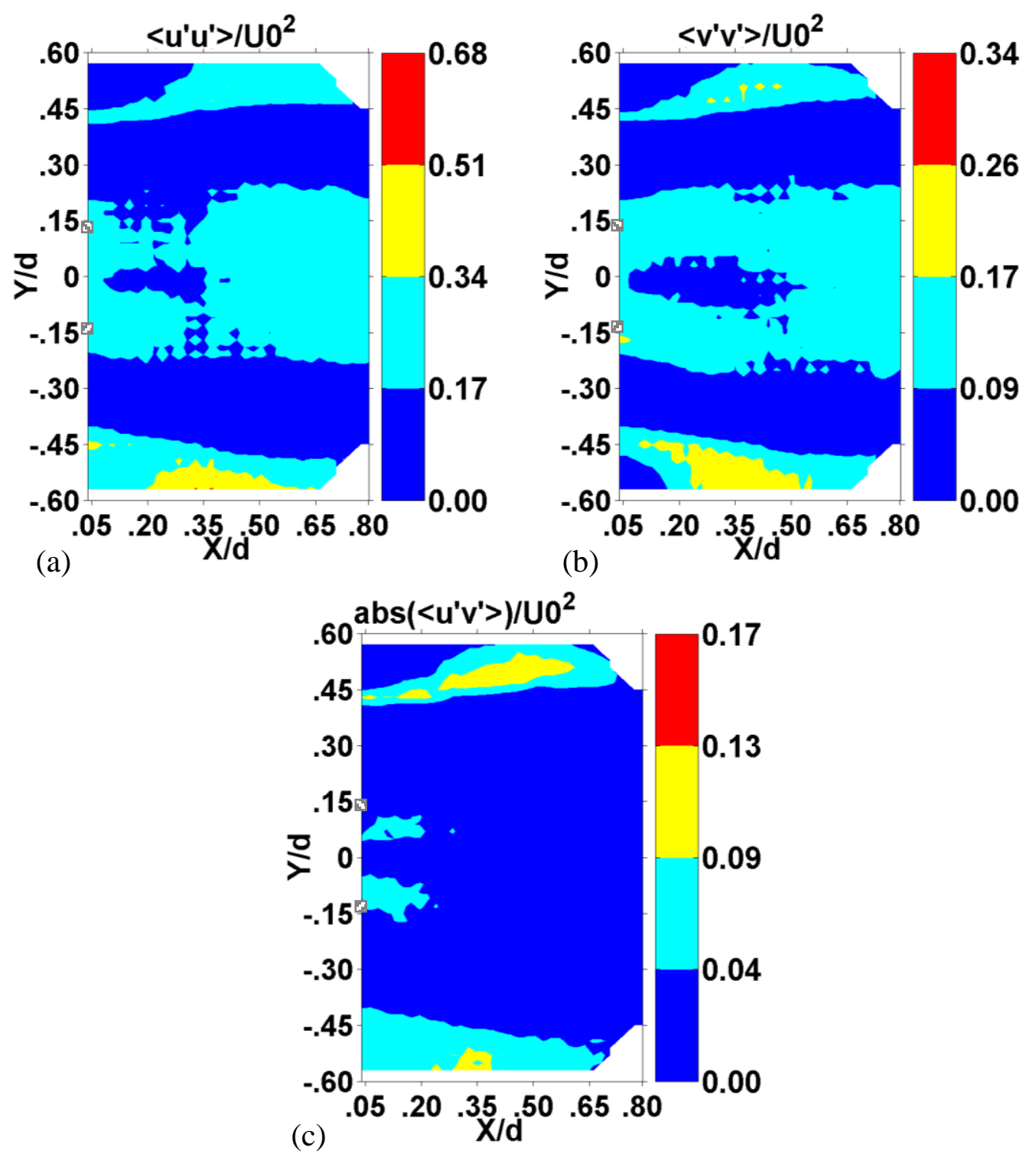

Figure 6. Contour plots of Reynolds stresses (a) $\left\langle u^{\prime} u^{\prime}\right\rangle / U_{0}^{2}$, (b) $\left\langle v^{\prime} v^{\prime}\right\rangle / U_{0}^{2}$, and $\left\langle u^{\prime} v^{\prime}>/ U_{0}^{2}\right.$. Leaflet tips were located at $Y / d \pm 0.14$ and $X / d=0$, and are shown as hatched boxes on the plots 

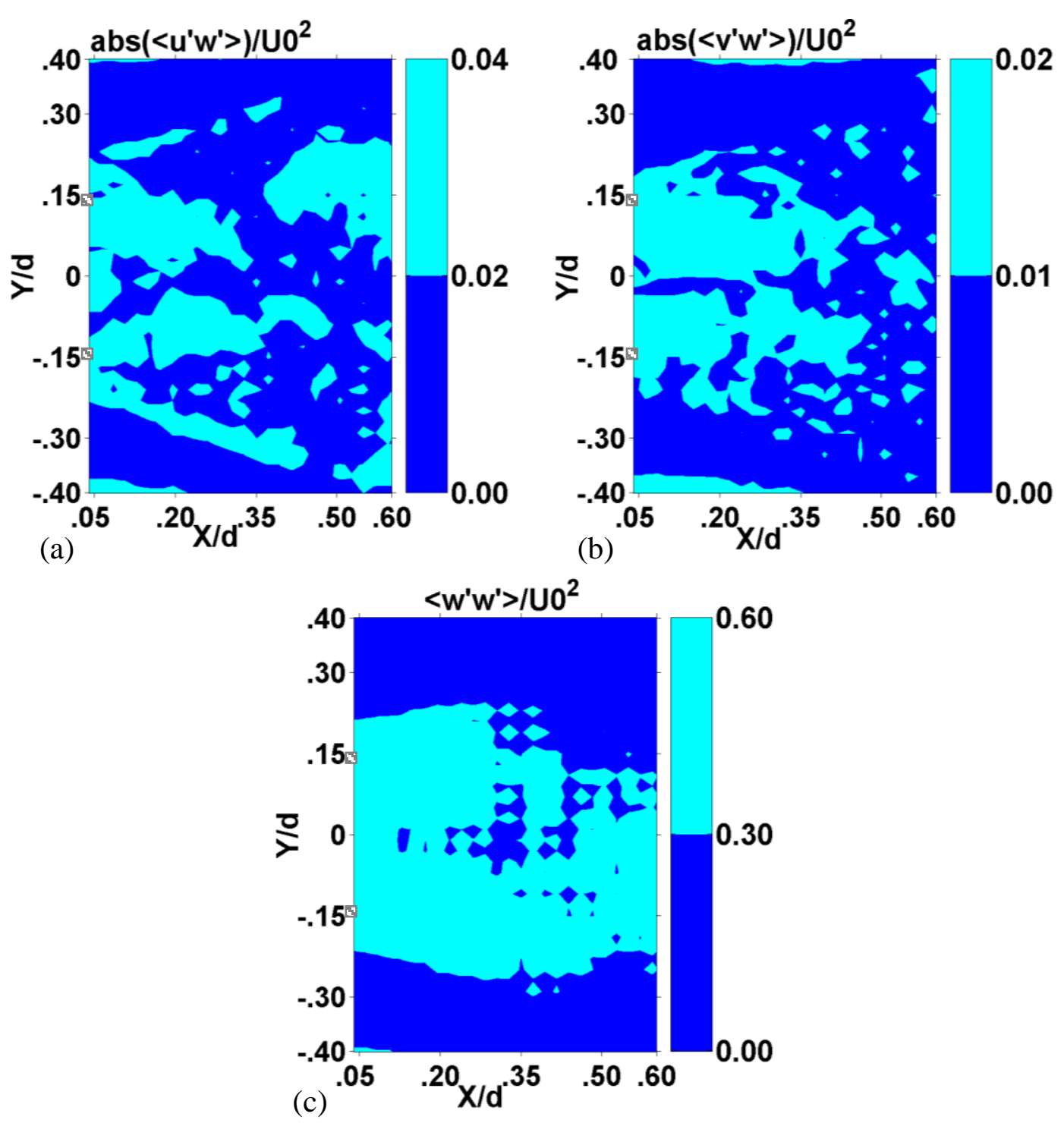

Figure 7. Contour plots of Reynolds stresses containing the out-of-plane turbulent velocity, $w^{\prime}$, (a) $\left\langle u^{\prime} w^{\prime}\right\rangle / U_{0}{ }^{2}$, (b) $\left\langle v^{\prime} w^{\prime}\right\rangle / U_{0}{ }^{2}$, and (c) $\left\langle w^{\prime} w^{\prime}>/ U_{0}{ }^{2}\right.$. Leaflet tips were located at $Y / d \pm 0.14$ and $X / d=0$, and are shown as hatched boxes on the plots 